\title{
KLV und Psychotherapien: Partnerschaft oder Verdächtigungen?
}

Das Bundesamt für Gesundheit ist zur Zeit daran, Artikel 2 und 3 der KLV [1] völlig zu überarbeiten. Diese Bestimmungen enthalten die Voraussetzungen der Leistungsvergütung für die drei wissenschaftlich anerkannten psychotherapeutischen Modelle. Falls der neue Text von der Eidgenössischen Leistungskommission am 4. Mai angenommen wird, tritt er am 1. Juli in Kraft [2]. Man versichert uns, dass damit die psychotherapeutischen Leistungen besser von den integrierten psychiatrischen Leistungen abgegrenzt, die Indikationen verbessert, die Kosten gesenkt und die Anzahl der «Missbräuche» [3] bei Psychotherapien verringert werden.

Artikel 2, der sich mit den Grundvoraussetzungen für die Rückerstattung der Psychotherapien befasst, verlangt zu Recht, dass die Wirksamkeit der angewandten Methode wissenschaftlich bewiesen sein muss. Im neuen Absatz 2 wird die Rückerstattung mit dem genauen Inhalt der Psychotherapie verbunden: ein Gegenstand (Krankheit[en]), ein Mittel (mindestens die Sprache), ein Prozess (zwischen dem Psychotherapeuten und dem Patienten), eine Psychopathologie (Diagnose), ein Rahmen (feste Sitzungen) und ein Ziel. Artikel 3 schliesst die Rückerstattung von Psychotherapien aus, die nicht aus Krankheitsgründen durchgeführt werden. Der Reifungsprozess der Persönlichkeit von Kindern und Jugendlichen bleibt jedoch erhalten und geschützt.

Mit Artikel 2 wird die Rückerstattung einer qualitativ hochwertigen psychotherapeutischen Leistung für alle Partner klar verteidigt [4]. Im Gegensatz dazu geht jedoch der neue Artikel 3 hauptsächlich auf die Interessen der Versicherer ein und entspricht nicht dem Grundsatz einer gemeinsam getragenen Verantwortung. Absatz 1 ist nicht auf ein allgemeines Interesse an der psychischen Volksgesundheit ausgerichtet, deren Probleme wir alle kennen, sondern auf Verdächtigungen: Die Patienten missbrauchen angeblich die Psychotherapie, und die Leistungsträger stellen missbräuchliche Indikationen oder sind inkompetent. In Absatz 1 wird die maximale Häufigkeit und Dauer der vom Versicherer vergüteten Psychotherapie nicht mehr geregelt. Statt dessen enthält er Angaben über die Beaufsichtigung der per definitionem verdächtigen Psychotherapeuten durch die Versicherer. An dieser Stelle gleitet die Verordnung, die eigentlich die Rückerstattung der Versicherer an ihre Kunden regeln sollte, in eine unangemessene Reglementierung der Beziehung zwischen Versicherern und Ärzten ab. Beweis dafür sind die von den Versicherungen in immer kürzeren Abständen geforderten Berichte. Dasselbe gilt für die Klarstellung, dass der Versicherer seine Entscheidung dem Leistungsträger mit Kopie an den Versicherten mitteilt. Eigentlich sollte es genau umgekehrt sein (Kopie an den Arzt), da der Versicherer dem Versicherten die Rückerstattung garantiert, nicht dem Arzt, dem der Patient die Rechnung zahlt. Mit diesem Absatz wird sich die Inanspruchnahme der Leistungen folglich in keinerlei Weise verbessern. Im Gegenteil: Es ist ein leichtes, die Zusatzkosten, die nicht offen ausgesprochene Absicht zum Bremsen der Indikationen für längere Behandlungen und die Stigmatisierung der psychiatrischen Behandlungen aufzuzeigen. Der fragliche Absatz vermischt die verschiedenen Stufen der Beziehung zwischen dem Versicherer und dem Versicherten bzw. dem Leistungsträger. Er enthält auch einen schalen Beigeschmack der Verdächtigung, der das Verhältnis zwischen den Partnern verändert und vor allem den Versicherten/Patienten schadet. Ferner bedauern wir, dass im Text keine Rekursinstanz für Streitfälle zwischen Patienten und Versicherern vorgesehen ist. Diese Bestimmung muss daher unbedingt umformuliert werden.

Im Unterschied zum ersten Absatz trägt der zweite dem Interesse der psychischen Volksgesundheit Rechnung. Er sieht die Erstellung eines Berichts nach 40 Sitzungen vor. Dieser Bericht muss einen durch Argumente untermauerten Vorschlag für die Fortsetzung der Psychotherapie anhand der in Artikel 2 festgelegten Kriterien umfassen. Wir halten es für unstrittig, dass ein solcher Bericht nach 40 Sitzungen im Interesse aller Partner (Patienten, Versicherer, Ärzte und Politik) ist.

Schliesslich ist die Forderung nach Beteiligung durch bessere Information über unsere Praxis ein legitimes Anliegen aller unserer Partner. Trotz der Vorurteile einiger dieser Partner arbeitet die Ärzteschaft unablässig daran und setzt sich für Qualität und einen Zugang zu einer Behandlung ein, die auf einer wissenschaftlichen Tätigkeit und einer fallweisen klinischen Betrachtung basiert. Eine auf dem Verdacht des Missbrauchs von Leistungen durch die Patienten und missbräuchlichen Indikationen von Ärzten aufgebaute Verordnung kann sie jedoch weder befürworten noch akzeptieren. Sollte dies die Ausrichtung sein, die das BAG der Revision des Leistungskatalogs geben will, würden wir uns mit aller Kraft zur Wehr setzen. Wir hoffen, dass das BAG auf die hier genannten Punkte achten wird, um die Bereinigungsarbeit so durchzuführen, dass alle Partner ihre Verantwortung wahrnehmen. Auf dem Gebiet der psychischen Volksgesundheit setzt dies voraus, dass Psychotherapien auf der Grundlage der Klinik einer psychischen Erkrankung und nicht einer Ideologie der allgemeinen Verdächtigung verordnet werden können.

Dr. med. Ursula Steiner-König, Vizepräsidentin der FMH

Dr. med. René Raggenbass,

Mitglied des Zentralvorstands der FMH

1 Verordnung des EDI vom 29. September 1995 über Leistungen in der obligatorischen Krankenpflegeversicherung (Krankenpflege-Leistungsverordnung, KLV).

2 Die entsprechenden Massnahmen sind auf den 31. Dezember 2010 befristet. Bis dahin wird das BAG die Wirkung zusammen mit den Versicherern und Leistungsträgern evaluieren.

3 Im Zuge der ersten Vernehmlassungen wurde die These des «Missbrauchs» psychotherapeutischer Leistungen und «falscher» Indikationen mehrmals vom BAG und von den Versicherern aufgestellt, aber nie objektiv untermauert. Folglich handelt es sich um reine Gerüchte.

4 Artikel 2 liegt folglich im direkten Interesse der Patienten (deren Behandlung bezahlt wird), der Versicherer (die wissen, für welche Leistungen sie bezahlen) und der Leistungsträger (Zugang zu psychotherapeutischen Leistungen über die Grundversicherung). 